\title{
Consistent house allocation
}

Citation for published version (APA):

Ehlers, L. H., \& Klaus, B. E. (2005). Consistent house allocation. METEOR, Maastricht University School of Business and Economics. METEOR Research Memorandum No. 008 https://doi.org/10.26481/umamet.2005008

Document status and date:

Published: 01/01/2005

DOI:

10.26481/umamet.2005008

Document Version:

Publisher's PDF, also known as Version of record

\section{Please check the document version of this publication:}

- A submitted manuscript is the version of the article upon submission and before peer-review. There can be important differences between the submitted version and the official published version of record.

People interested in the research are advised to contact the author for the final version of the publication, or visit the DOI to the publisher's website.

- The final author version and the galley proof are versions of the publication after peer review.

- The final published version features the final layout of the paper including the volume, issue and page numbers.

Link to publication

\footnotetext{
General rights rights.

- You may freely distribute the URL identifying the publication in the public portal. please follow below link for the End User Agreement:

www.umlib.nl/taverne-license

Take down policy

If you believe that this document breaches copyright please contact us at:

repository@maastrichtuniversity.nl

providing details and we will investigate your claim.
}

Copyright and moral rights for the publications made accessible in the public portal are retained by the authors and/or other copyright owners and it is a condition of accessing publications that users recognise and abide by the legal requirements associated with these

- Users may download and print one copy of any publication from the public portal for the purpose of private study or research.

- You may not further distribute the material or use it for any profit-making activity or commercial gain

If the publication is distributed under the terms of Article $25 \mathrm{fa}$ of the Dutch Copyright Act, indicated by the "Taverne" license above, 


\title{
Consistent House Allocation*
}

\author{
Lars Ehlers ${ }^{\dagger} \quad$ Bettina Klaus ${ }^{\ddagger}$
}

October 2005

\begin{abstract}
In practice we often face the problem of assigning indivisible objects (e.g., schools, housing, jobs, offices) to agents (e.g., students, homeless, workers, professors) when monetary compensations are not possible. We show that a rule that satisfies consistency, strategy-proofness, and efficiency must be an efficient generalized priority rule; i.e., it must adapt to an acyclic priority structure, except-maybe - for up to three agents in each object's priority ordering. JEL Classification: D63, D70

Keywords: indivisible objects, priority structure, consistency, strategy-proofness.
\end{abstract}

\section{Introduction}

In real life we often face the problem of allocating heterogeneous indivisible objects (for instance, schools, housing, jobs, or offices) among a group of agents (for instance, students, homeless, workers, or professors) when monetary compensations are not possible. Agents have strict preferences over objects and remaining unassigned. An assignment is an allocation of the objects to the agents such that every agent receives at most one object. A rule is a systematic way of solving these assignment problems that are classically called house allocation problems (the implicit assumption being that each agent only needs at most one house to live in). The search for "good" house allocation rules, i.e., rules with desirable properties, is the subject of many recent papers, for instance, Abdulkadiroğlu and Sönmez (1998, 1999), Bogomolnaia and Moulin (2001), Chambers (2004), Ehlers (2002), Ehlers and Klaus (2003,2005), Ehlers, Klaus, and Pápai (2002), Ergin (2000), Kesten (2004a,b), Pápai (2000), and Svensson (1999). Recent articles that pursue a similar research agenda in closely related models with monetary compensations are Miyagawa (2001), Ohseto (2006), Schummer (2000), Svensson (2004), Svensson and Larsson (2002), and Thomson (2003). ${ }^{1}$

In most real life problems "priorities" naturally arise. For example, in school choice students who live closer to a school and/or have siblings attending a school have higher priority to be admitted at that school (Abdulkadiroğlu and Sönmez, 2003). When apartments are allocated,

\footnotetext{
${ }^{*}$ We are grateful to the Editor and an anonymous referee for helpful comments and suggestions.

${ }^{\dagger}$ Département de Sciences Économiques and CIREQ, Université de Montréal, Montréal, Québec H3C 3J7, Canada; e-mail: lars.ehlers@umontreal.ca. L. Ehlers acknowledges financial support from the SSHRC (Canada).

${ }^{\ddagger}$ Corresponding Author: Department of Economics, Maastricht University, P.O. Box 616, 6200 MD Maastricht, The Netherlands; e-mail: B.Klaus@algec.unimaas.nl.

${ }^{1}$ This list is not exhaustive.
} 
the homeless who have been waiting longer have higher priority to be assigned an apartment (similarly for the placement of students at colleges).

Balinski and Sönmez (1999) were the first to formulate the assignment problem based on priorities. The agents' priorities for a certain object are captured by an ordering of the agents. A priority structure is a profile specifying for each object a priority ordering. Given the agents' priorities, it is natural to require that the assignment does not violate any priorities. This means that there should be no agent who - conditional on higher priority - envies another agent (for receiving a better object). A rule adapts to a priority structure if it always chooses an assignment that does not violate any priorities.

It is well known, that for any profile of agents' preferences the assignment obtained from applying Gale and Shapley's (1962) deferred acceptance algorithm Pareto dominates any other assignment which does not violate any priorities. This algorithm is called the "best" rule among the rules adapting to a priority structure. Unfortunately the best rule may not be efficient. Ergin (2002) shows that "acyclicity" of the priority structure is equivalent to various properties (efficiency, group strategy-proofness, ${ }^{2}$ and consistency) of the induced best rules.

Consistency, our main property, is a condition of stability when the set of agents and resources may change. To understand this property, suppose that after objects are allocated according to a rule, some agents leave the economy with their allotments, and the remaining agents "reassign" among themselves the remaining objects. What if the same rule is applied to their "reassignment problem"? A rule is considered "unstable" or "inconsistent" if its reassignment differs from its original assignment to the remaining agents. ${ }^{3}$

Our main result shows that a rule that satisfies consistency, strategy-proofness, ${ }^{4}$ and efficiency must be an efficient generalized priority rule; i.e., it is efficient and adapts to an acyclic priority structure, except - maybe - for up to three agents in each object's priority ordering. Therefore, our properties imply that the assignment of objects must be based on an acyclic generalized priority structure and the rule chooses the same allocations as the associated deferred acceptance algorithm.

We proceed as follows. In Section 2 we introduce the model and the main properties. Section 3 we devote to (generalized) priority rules, two examples, and the main result. We conclude in Section 4 with a brief discussion of our results and a comparison to Ehlers and Klaus (2005). The proof of the main result is given in the Appendix.

\section{House Allocation}

Let $P$ denote the set of potential agents. We assume that $P$ is finite and contains at least four agents. ${ }^{5}$ Let $\mathcal{P}$ denote the set of all subsets of $P$ containing at least two agents. Let $K$ denote the set of potential real objects. The set $K$ can be finite or infinite. Not receiving any real

\footnotetext{
${ }^{2}$ By group strategy-proofness no group of agents can profit by joint misrepresentation of their preferences such that all members of the group weakly gain and at least one member of the group strictly gains.

${ }^{3}$ Ergin (2000) studies consistency for the house allocation problem in various combinations with efficiency, converse consistency, neutrality, and anonymity. Here, converse consistency pertains to the opposite operation of consistency; see Thomson (2004). By neutrality, the names of the objects do not matter. By anonymity, the names of the agents do not matter.

${ }^{4}$ No agent can manipulate the allocation to his/her advantage by lying about his/her preferences.

${ }^{5}$ Example 2 considers $|P|=3$ and clarifies why our main result has "no bite" in this case. Our results remain unchanged if $P$ is infinite.
} 
object is called "receiving the null object." The null object, denoted by 0 , does not belong to $K$ and is available in any economy. Let $\mathcal{H}$ denote the set of all finite subsets of $K$.

Each agent $i \in P$ is equipped with a strict preference relation $R_{i}$ over all objects $K \cup\{0\}$. In other words, $R_{i}$ is a linear order over $K \cup\{0\}$. Given $x, y \in K \cup\{0\}, x P_{i} y$ means that agent $i$ strictly prefers $x$ to $y$ under $R_{i}$. Let $\mathcal{R}$ denote the set of all linear orders over $K \cup\{0\}$. Given $N \subseteq P$, let $\mathcal{R}^{N}$ denote the set of all (preference) profiles $R=\left(R_{i}\right)_{i \in N}$ such that for all $i \in N$, $R_{i} \in \mathcal{R}$. Given $N \subseteq P, i \in N, R \in \mathcal{R}^{N}$, and $K^{\prime} \subseteq K \cup\{0\}$, let $R_{i \mid K^{\prime}}$ denote the restriction of $R_{i}$ to $K^{\prime}$ and $R_{\mid K^{\prime}}=\left(R_{i \mid K^{\prime}}\right)_{i \in N}$. An economy (or house allocation problem) consists of a set of agents, their preferences, and a finite set of real objects which have to be allocated among them. Formally, an economy is a triple $(N, R, H)$ where $N \in \mathcal{P}, R \in \mathcal{R}^{N}$, and $H \in \mathcal{H}$. We suppress the set of agents and write $(R, H)$ instead of $(N, R, H)$. Let $\mathcal{E}^{N}$ denote the set of all economies with the set of agents equal to $N$.

When allocating objects each agent receives one object. The null object is the only object which can be assigned to several agents. Formally, given a set of agents $N$, an allocation is a list $a=\left(a_{i}\right)_{i \in N}$ such that for all $i \in N, a_{i} \in K \cup\{0\}$, and none of the real objects in $K$ is assigned to more than one agent. Note that not all real objects in $K$ have to be assigned. An (allocation) rule is a function that assigns an allocation to every economy. Formally, a rule $\varphi$ chooses for all $N \in \mathcal{P}$ and all economies $(R, H) \in \mathcal{E}^{N}$ an allocation $\varphi(R, H)$ such that for all $i \in N, \varphi_{i}(R, H) \in H \cup\{0\}$. Given $i \in N$, we call $\varphi_{i}(R, H)$ the allotment of agent $i$ at $\varphi(R, H)$.

Next, we introduce our main properties for rules.

First, the rule chooses only (Pareto) efficient allocations.

Efficiency: For all $N \in \mathcal{P}$ and all $(R, H) \in \mathcal{E}^{N}$, there is no allocation $a=\left(a_{i}\right)_{i \in N}$ such that for all $i \in N, a_{i} \in H \cup\{0\}$ and $a_{i} R_{i} \varphi_{i}(R)$, and for some $j \in N, a_{j} P_{j} \varphi_{j}(R, H)$.

Given $N \in \mathcal{P}, R \in \mathcal{R}^{N}$, and $M \subseteq N$, let $R_{M}$ denote the profile $\left(R_{i}\right)_{i \in M}$. It is the restriction of profile $R$ to the subset of agents $M$. We also use the notation $R_{-i}=R_{N \backslash\{i\}}$. For example, $\left(\bar{R}_{i}, R_{-i}\right)$ denotes the profile obtained from $R$ by replacing $R_{i}$ by $\bar{R}_{i}$.

Second, no agent ever benefits from misrepresenting his/her preference relation.

Strategy-Proofness: For all $N \in \mathcal{P}$, all $(R, H) \in \mathcal{E}^{N}$, all $i \in N$, and all $\bar{R}_{i} \in \mathcal{R}, \varphi_{i}(R, H) R_{i}$ $\varphi_{i}\left(\left(\bar{R}_{i}, R_{-i}\right), H\right)$.

Our last property is a stability condition. Consistency ${ }^{6}$ requires that if some agents leave an economy with their allotments, then the rule should allocate the remaining objects among the agents who did not leave in the same way as in the original economy.

Consistency: For all $N, M \in \mathcal{P}$ such that $M \subseteq N$, all $(R, H) \in \mathcal{E}^{N}$, and all $i \in M$,

$$
\varphi_{i}(R, H)=\varphi_{i}\left(R_{M}, H \backslash\left(\cup_{j \in N \backslash M}\left\{\varphi_{j}(R, H)\right\}\right)\right) .
$$

Remark 1. Strategy-proofness and consistency imply that only preferences over objects that are present in an economy matter for the final allocation. Formally, for any strategy-proof and consistent rule $\varphi$, if two economies $(N, R, H)$ and $\left(N, R^{\prime}, H\right)$ are such that $R_{\mid H \cup\{0\}}=R_{\mid H \cup\{0\}}^{\prime}$, then $\varphi(R, H)=\varphi\left(R^{\prime}, H\right){ }^{7}$

\footnotetext{
${ }^{6}$ For a recent overview see Thomson (2004).

${ }^{7}$ Without loss of generality, let $R^{\prime}=\left(R_{i}^{\prime}, R_{-i}\right)$ for some $i \in N$. By strategy-proofness and $R_{i \mid H \cup\{0\}}=R_{i \mid H \cup\{0\}}^{\prime}$, we obtain $\varphi_{i}(R, H)=\varphi_{i}\left(R^{\prime}, H\right)$. Thus, by consistency and $\left(R_{N \backslash\{i\}}, H \backslash\left\{\varphi_{i}(R, H)\right\}\right)=\left(R_{N \backslash\{i\}}^{\prime}, H \backslash\left\{\varphi_{i}\left(R^{\prime}, H\right)\right\}\right)$, we have for all $j \in N \backslash\{i\}, \varphi_{j}(R, H)=\varphi_{j}\left(R_{N \backslash\{i\}}, H \backslash\left\{\varphi_{i}(R, H)\right\}\right)=\varphi_{j}\left(R^{\prime}, H\right)$. Hence, $\varphi(R, H)=\varphi\left(R^{\prime}, H\right)$.
} 


\section{Generalized Priority Rules}

We now introduce the idea that rules may adapt to priorities. In many situations "priorities" naturally arise. For example, when offices are assigned to the members of a department, seniority may matter; when apartments are assigned to graduate students, students who have been waiting longer should come first; and for jobs, a candidate with higher qualification may be ranked above lower qualified applicants.

We follow the notation and terminology introduced by Ergin (2002), who studies efficient house allocation on the basis of priorities.

Given $x \in K$, let $\succ_{x}$ denote a linear order over $P$. We call $\succ_{x}$ a priority ordering for object $x$. A priority structure is a profile $\succ=\left(\succ_{x}\right)_{x \in K}$ specifying for each object a priority ordering. Given $N \in \mathcal{P}, i \in N, R \in \mathcal{R}^{N}, x \in K$, and a priority structure $\succ$, an allocation $a$ violates the priority of $i$ for $x$ if there exists $j \in N$ such that $a_{j}=x, i \succ_{x} j$, and $x P_{i} a_{i}$ (i.e., $i$ has higher priority for object $x$ than $j$ but $j$ receives $x$ and $i$ envies $j$ ). A rule $\varphi$ adapts to $a$ priority structure $\succ$ if for all $N \in \mathcal{P}$ and all $(R, H) \in \mathcal{E}^{N}, \varphi(R, H)$ does not violate the priority of any agent for any object.

We say that $\varphi$ is a priority rule if there exists a priority structure $\succ$ such that $\varphi$ adapts to $\succ$.

Using a result from Balinski and Sönmez (1999, Theorem 2) it follows that an efficient priority rule $\varphi$ must be a so-called best rule; i.e., for each economy $(R, H) \in \mathcal{E}^{N}, \varphi(R, H)$ is obtained from applying the agents-proposing deferred acceptance algorithm (Gale and Shapley, 1962) to the two-sided matching problem where the agents' preferences are given by $R$ and the "objects' preferences" are given by the priority structure $\succ$ (see also Ergin, 2002, Proposition 1). Ergin's (2002, Theorem 1) main result essentially states that for best rules, efficiency, group strategy-proofness, consistency, and the acyclicity of the priority structure are all equivalent.

While Ergin (2002) focuses on the class of rules that adapt to an exogenously given priority structure, we consider the general class of all rules. We show that if a rule satisfies consistency, strategy-proofness, and efficiency, then there must exist a priority structure that it "almost" adapts to. In order to formalize this "almost" adaptation, we introduce generalized priority rules next.

Let $x \in K$. We call a binary relation $\succ_{x}$ a generalized priority ordering if

(i) $\succ_{x}$ is transitive and antisymmetric and

(ii) there exists a set $Q^{x} \subseteq P$ such that $\left|Q^{x}\right| \leq 3$ and

(a) the restriction of $\succ_{x}$ to the agents in $P \backslash Q^{x}$, denoted $\succ_{x \mid P \backslash Q^{x}}$, is complete (all agents in $P$ except for the agents in $Q^{x}$ can be completely ranked according to priority order $\succ_{x}$ ) and

(b) for all $i \in P \backslash Q^{x}$ and all $j \in Q^{x}, i \succ_{x} j$ (agents in $P \backslash Q^{x}$ are ranked above agents in $Q^{x}$ ).

Note that any priority ordering is a generalized priority ordering since it satisfies the above definition for $Q^{x}=\emptyset$. Also, a generalized priority ordering that satisfies the above definition with $\left|Q^{x}\right|=1$ is in fact a priority ordering. 
A generalized priority structure is a profile $\succ=\left(\succ_{x}\right)_{x \in K}$ specifying for each object a generalized priority ordering. We say that $\varphi$ is a generalized priority rule if there exists a generalized priority structure $\succ$ such that $\varphi$ adapts to $\succ$; i.e., it adapts to all priorities that are specified in the generalized priority ordering, except possibly for priorities concerning an object $x$ and agents in $Q^{x}$.

Theorem 1. If a rule satisfies consistency, strategy-proofness, and efficiency, then it is an efficient generalized priority rule.

In order to be more specific about the structure of efficient generalized priority rules, we use Ergin's (2002, Theorem 1) result that a priority rule is efficient if and only if the underlying priority structure is acyclic.

Let $\succ$ be a generalized priority structure. We say that $\succ$ has a cycle if there exist $x, y \in K$ and $i, j, k \in P$ such that $i \succ_{x} j \succ_{x} k \succ_{y} i$. A generalized priority structure is acyclic if it has no cycles. We can now rephrase Theorem 1 .

Corollary 1. If a rule satisfies consistency, strategy-proofness, and efficiency, then it adapts to an acyclic generalized priority structure $\succ$.

Efficient priority rules satisfy all the axioms in Theorem 1. In the following example, we demonstrate that the class of efficient generalized priority rules is strictly larger than the class of efficient priority rules. In order to do so, we need some notation.

Let $\succ$ be a priority structure and $\varphi$ be a priority rule adapting to $\succ$. Then we call $\varphi$ a serial dictatorship if there exists an ordering $\succ_{*}$ over $P$ such that for all $x \in K, \succ_{x}=\succ_{*}$. We denote by $\varphi^{\succ *}$ the serial dictatorship with ordering $\succ_{*}$. It is easily checked that a serial dictatorship satisfies consistency, strategy-proofness, and efficiency.

Example 1. Let $\succ_{*}^{1}$ and $\succ_{*}^{2}$ be the following orderings over $P$ :

$$
\begin{aligned}
& |P| \succ_{*}^{1}|P|-1 \succ_{*}^{1} \cdots \succ_{*}^{1} 3 \succ_{*}^{1} 2 \succ_{*}^{1} 1 ; \\
& |P| \succ_{*}^{2}|P|-1 \succ_{*}^{2} \cdots \succ_{*}^{2} 3 \succ_{*}^{2} 1 \succ_{*}^{2} 2 .
\end{aligned}
$$

The orderings $\succ_{*}^{1}$ and $\succ_{*}^{2}$ only differ in their last two entries. We define the following, "almost serial dictatorship:" Agents start choosing their objects according to their names, the agent with the highest number starts and chooses his/her favorite object, then the agents with the next higher number chooses his/her favorite object among the remaining objects, etc., until only agents 1 and 2 are left over. Now, the number of remaining objects determines who is allowed to choose next. For all $N \in \mathcal{P}$ and all $(R, H) \in \mathcal{E}^{N}$,

(i) if $\left|H \backslash\left(\cup_{i \in N \backslash\{1,2\}}\left\{\varphi_{i}^{\succ^{1}}(R, H)\right\}\right)\right| \leq 1$, then $\varphi(R, H) \equiv \varphi^{\succ^{*}}(R, H)$ (if one or none object are left for agents 1 and 2 , then agent 2 may choose among the remaining objects before agent 1); and

(ii) if $\left|H \backslash\left(\cup_{i \in N \backslash\{1,2\}}\left\{\varphi_{i}^{\succ^{*}}(R, H)\right\}\right)\right|>1$, then $\varphi(R, H) \equiv \varphi^{\succ^{2}}(R, H)$ (if more than one object is left for agents 1 and 2, then agent 1 may choose among the remaining objects before agent 2). 
Because serial dictatorships are efficient, the rule $\varphi$ is efficient. Since agents 1 and 2 cannot change the ordering by stating other preferences and $\succ_{*}^{1}$ and $\succ_{*}^{2}$ only differ in their last two entries, the rule $\varphi$ is strategy-proof. To show consistency let $N \in \mathcal{P},(R, H) \in \mathcal{E}^{N}$, and $j \in N$. It suffices to show that for all $i \in N \backslash\{j\}$,

$$
\varphi_{i}\left(R_{-j}, H \backslash\left\{\varphi_{j}(R, H)\right\}\right)=\varphi_{i}(R, H) .
$$

If $i \quad \in \quad N \backslash\{1,2\}$, then $\varphi_{i}^{\succ *}(R, H)=\varphi_{i}^{\succ^{2}}(R, H)$ and $\varphi_{i}^{\succ^{*}}\left(R_{-j}, H \backslash\left\{\varphi_{j}(R, H)\right\}\right)=$ $\varphi_{i}^{\succ}{ }^{2}\left(R_{-j}, H \backslash\left\{\varphi_{j}(R, H)\right\}\right)$. So, the fact that $\varphi$ is a serial dictatorship for all agents except agents 1 and 2 implies (1).

Consider $i \in\{1,2\}$ and denote $\{1,2\}=\{i, k\}$. If either $j=k$ or $k \notin N$, then $\varphi_{i}^{\succ *}\left(R_{-j}, H \backslash\left\{\varphi_{j}(R, H)\right\}\right)=\varphi_{i}^{\succ^{2}}\left(R_{-j}, H \backslash\left\{\varphi_{j}(R, H)\right\}\right)$. Since either $\varphi(R, H)=\varphi^{\succ^{*}}(R, H)$ or $\varphi(R, H)=\varphi^{\succ^{2}}(R, H)$, the consistency property of $\varphi^{\succ *}$ or $\varphi^{\succ_{*}^{2}}$ implies (1).

Finally, consider $i \in\{1,2\}$ and $j \in N \backslash\{1,2\} . \quad$ For all $i \in$ $N \backslash\{1,2\}, \quad \varphi_{i}^{\succ *}\left(R_{-j}, H \backslash\left\{\varphi_{j}^{\succ^{1}}(R, H)\right\}\right)=\varphi_{i}^{\succ^{*}}(R, H) \quad$ and $\quad \varphi_{j}(R, H)=$ $\varphi_{j}^{\succ^{1}}(R, H)$. Thus, $\quad\left(H \backslash\left\{\varphi_{j}(R, H)\right\}\right) \backslash\left(\cup_{i \in N \backslash\{1,2\}}\left\{\varphi_{i}^{\succ^{1}}\left(R_{-j}, H \backslash\left\{\varphi_{j}^{\succ^{*}}(R, H)\right\}\right)\right\}\right)=$ $\left(H \backslash\left\{\varphi_{j}^{\succ^{-1}}(R, H)\right\}\right) \backslash\left(\cup_{i \in N \backslash\{1,2\}}\left\{\varphi_{i}^{\succ^{1}}(R, H)\right\}\right)=H \backslash\left(\cup_{i \in N \backslash\{1,2\}}\left\{\varphi_{i}^{\succ^{1}}(R, H)\right\}\right)$.

Then either (i) holds for $(R, H)$ and $\left(R_{-j}, H \backslash\left\{\varphi_{j}^{\succ^{1}}(R, H)\right\}\right)\left(\varphi_{i}^{\succ *}\right.$ is used for both problems) or (ii) holds for $(R, H)$ and $\left(R_{-j}, H \backslash\left\{\varphi_{j}^{\succ *}(R, H)\right\}\right)\left(\varphi_{i}^{\succ^{*}}\right.$ is used for both problems). Hence, the consistency property of $\varphi^{\succ_{*}^{1}}$ or $\varphi^{\succ_{*}^{2}}$ implies (1). Finally note that $\varphi$ is a generalized priority rule where for all $x \in K, \succ_{x}=\succ_{*}^{1} \cap \succ_{*}^{2}$ and for all $x \in K, Q^{x}=\{1,2\}$.

Remark 2. In Example 1 the choice of $\succ_{*}^{1}$ or $\succ_{*}^{2}$ could be defined in a more complex manner without violating the axioms of Theorem 1 . We could choose $\succ_{*}^{1}$ whenever a certain object $x$ is available after the other agents (except agents 1 and 2) have received their allotments and when $x$ is not available, then we pick $\succ_{*}^{2}$. On the other hand, we can easily define efficient generalized priority rules that do not satisfy the axioms of Theorem 1; for instance if the choice between $\succ_{*}^{1}$ and $\succ_{*}^{2}$ depends on the preferences of agent 1 (possible violations of strategy-proofness) or if the choice between $\succ_{*}^{1}$ and $\succ_{*}^{2}$ depends on the presence of certain agents (possible violations of consistency). Because it is intuitively clear, what the degrees of freedom in difference to the class of priority rules is, but since, at the same time, it is very tedious and technical to give a full characterization, we did not try to formulate Theorem 1 as a full characterization. The important point is that any rule satisfying the axioms of Theorem 1 is "almost" a priority rule. $\triangleleft$

Remark 3. Theorem 1 and Example 1 show that consistency, strategy-proofness and efficiency characterize "almost" efficient priority rules. These axioms only allow more flexibility at the bottom of the priority orderings-up to three agents for each object's generalized priority ordering. A similar feature has been observed in the paper by Bogomolnaia, Deb and Ehlers (2005). They show on the domain of weak preference relations that non-bossiness, ${ }^{8}$ strategy-proofness, and efficiency "almost" characterize serial dictatorships. Those axioms only allow more flexibility for the first two agents of the serial order.

\footnotetext{
${ }^{8}$ No agent can influence another agent's final allotment without changing his/her final consumption.
} 
In Example 1 the set of agents for which priorities are unspecified is equal to $\{1,2\}=$ $\cup_{x \in K} Q^{x}$. Next, we construct an efficient, strategy-proof, and consistent rule for $P=\{1,2,3\}$ and $K=\{a, b\}$ that does not adapt to any priority structure. Extending the example to $|P|>3$, as explained after the example, yields an example where the set of agents for which priorities are unspecified is equal to $\{1,2,3\}=\cup_{x \in K} Q^{x}$.

Example 2. Let $P=\{1,2,3\}$ and $K=\{a, b\}$. The rule $\bar{\varphi}$ is defined as follows:

For $N=\{i, j, k\}$ such that $|N|=3$ define $\varphi^{i j k}$ as the serial dictatorship $\varphi^{\succ}$ where $i \succ_{a} j \succ_{a} k$ and $i \succ_{b} j \succ_{b} k$.

- If $|N|=3, H=\{a, b\}$, and $R \in \mathcal{R}^{N}$ such that

$-\varphi_{1}^{132}(R, H)=a$ and $\varphi_{3}^{132}(R, H)=b$, then $\bar{\varphi}(R, H)=\varphi^{132}(R, H)$,

- $\varphi_{1}^{132}(R, H)=a$ and $\varphi_{3}^{132}(R, H)=0$, then $\bar{\varphi}(R, H)=\varphi^{213}(R, H)$,

- $\varphi_{1}^{123}(R, H)=b$ and $\varphi_{2}^{123}(R, H)=a$, then $\bar{\varphi}(R, H)=\varphi^{123}(R, H)$,

- $\varphi_{1}^{123}(R, H)=b$ and $\varphi_{2}^{123}(R, H)=0$, then $\bar{\varphi}(R, H)=\varphi^{312}(R, H)$.

- If $H=\{a\}$ and $R \in \mathcal{R}^{N}$, then $\bar{\varphi}(R, H)=\varphi^{123}(R, H)$.

- If $H=\{b\}$, and $R \in \mathcal{R}^{N}$, then $\bar{\varphi}(R, H)=\varphi^{132}(R, H)$.

For $N=\{1,2\}$ let $\succ^{[12]}$ denote the priority structure $2 \succ_{a}^{[12]} 1$ and $1 \succ_{b}^{[12]} 2$.

- If $N=\{1,2\}, H=\{a, b\}$, and $R \in \mathcal{R}^{N}$, then $\bar{\varphi}(R, H)=\varphi^{\succ^{[12]}}(R, H)$.

For $N=\{1,3\}$ let $\succ^{[13]}$ denote the priority structure $1 \succ_{a}^{[13]} 3$ and $3 \succ_{b}^{[13]} 1$.

- If $N=\{1,3\}, H=\{a, b\}$, and $R \in \mathcal{R}^{N}$, then $\bar{\varphi}(R, H)=\varphi^{\succ^{[13]}}(R, H)$.

For $N=\{2,3\}$ let $\succ^{[23]}$ denote the priority structure $2 \succ_{a}^{[23]} 3$ and $3 \succ_{b}^{[23]} 2$.

- If $N=\{2,3\}, H=\{a, b\}$, and $R \in \mathcal{R}^{N}$, then $\bar{\varphi}(R, H)=\varphi^{\succ^{[23]}}(R, H)$.

- If $|N|=3, H=\{a, b\}$, and $R \in \mathcal{R}^{N}$ is such that the null object is the most preferred object under $R_{1}$, then $\bar{\varphi}(R, H)=\left(0, \varphi_{2}^{\succ}{ }^{[23]}\left(R_{\{2,3\}}, H\right), \varphi_{3}^{\succ}{ }^{[23]}\left(R_{\{2,3\}}, H\right)\right)$.

Since all rules used to define $\bar{\varphi}$ are priority rules, $\bar{\varphi}$ is efficient. The proof that $\bar{\varphi}$ satisfies strategy-proofness and consistency is straightforward, but tedious and is available from the authors upon request.

Example 2 demonstrates that at the bottom of each generalized priority ordering there may be a set of three agents which are not related to each other, i.e., $Q^{a}=Q^{b}=\{1,2,3\}$. Thus, the upper bound 3 for the cardinality of $Q^{x}$ cannot be lowered in the definition of a generalized priority ordering $\succ_{x}$. Furthermore, Example 2 can be easily adapted to any number of agents. Just let all other agents choose according to a serial dictatorship before agents $\{1,2,3\}$ and use then the rule of Example 2 to allocate objects to $\{1,2,3\}$, depending on which objects are left behind by the agents $N \backslash\{1,2,3\} .^{9}$

\footnotetext{
${ }^{9}$ In Example 2 the priority ordering of an object depends on which objects are available. Let $i \succ_{x}^{H} j$ mean that
} 


\section{Discussion}

Ehlers and Klaus (2005) consider an alternative consistency condition, called reallocationconsistency. In difference to consistency, this notion assumes that if some agents leave an economy with their allotments, then the rule should assign the objects in the same way to these agents for the economy where only the agents that left and their previous allotments are present.

Reallocation-Consistency: For all $N, M \in \mathcal{P}$ such that $M \subseteq N$, all $(R, H) \in \mathcal{E}^{N}$, and all $i \in M$,

$$
\varphi_{i}(R, H)=\varphi_{i}\left(R_{M}, \cup_{j \in M}\left\{\varphi_{j}(R, H)\right\}\right) .
$$

The difference between the two consistency properties is as follows. When defining consistency, we define the so-called reduced economy for consistent rules to equal the set of agents that were left behind and all objects not consumed by the agents that were leaving. Hence, in such a reduced economy there may be some unassigned objects in addition to the remaining agents' allotments - an incidence that cannot occur in a reduced economy for reallocation-consistent rules where agents can only reallocate their allotments among themselves. A priori, no logical relation exists between consistency and reallocation-consistency. Ehlers and Klaus (2005, Theorem 1) show that a rule satisfies reallocation-consistency, strategy-proofness, and efficiency if and only if it is an efficient priority rule. Since the set of efficient priority rules is a strict subset of the set of efficient generalized priority rules, reallocation-consistency - in combination with strategyproofness and efficiency - is a more demanding property than consistency. Hence, using the weaker (and more standard) condition of consistency here, yields a larger class of rules, but not a fully characterized set of rules.

This is also the reason why our Theorem 1 requires arguments that are different from Ehlers and Klaus (2005). In seeing why, fix a strategy-proof and efficient rule $\varphi$ and let $x \in K$ be an arbitrary object. An obvious way to derive a priority ordering for $x$ is the following: for all $i \in N$, let $R_{i}^{x} \in \mathcal{R}$ be such that for all $y \in K \backslash\{x\}, x P_{i}^{x} 0 P_{i}^{x} y$ (object $x$ is the only object which is preferred to the null object); then define

$$
i \succ_{x} j \Leftrightarrow \varphi_{i}\left(\left(R_{i}^{x}, R_{j}^{x}\right),\{x\}\right)=x .
$$

If $\varphi$ is reallocation-consistent, then it is easy to see that $\succ_{x}$ is a linear order and for all $H \in \mathcal{H}$ such that $x \in H$,

$$
i \succ_{x} j \Leftrightarrow \varphi_{i}\left(\left(R_{i}^{x}, R_{j}^{x}\right), H\right)=x .
$$

Hence, we could have also used $(*)$ to define $\succ_{x}$. If $\varphi$ is consistent, then it is still true that $\succ_{x}$ is a linear order but (*) may not be true. In Example 1, we have $2 \succ_{x} 1$ but $\varphi_{1}\left(\left(R_{1}^{x}, R_{2}^{x}\right), H\right)=x$ for all $H \in \mathcal{H}$ such that $\{x\} \subsetneq H$. The main difficulty of our proof is to show that for any set consisting of four agents, say $N=\{1,2,3,4\},(*)$ holds for the highest $\succ_{x}$-ranked agent in this set and any other agent in $N$, i.e., if agent 1 is the highest $\succ_{x}$-ranked agent in $N$, then $(*)$ holds for agents 1 and 2, for agents 1 and 3, and for agents 1 and 4. Once this is shown, the rest of our proof uses arguments similar to Ehlers and Klaus (2005).

\footnotetext{
$i$ has higher priority for object $x$ than $j$ when $H \cup\{0\}$ is the set of available objects. Then we have $1 \succ_{a}^{\{a\}} 2 \succ_{a}^{\{a\}} 3$ and $2 \succ_{a}^{\{a, b\}} 1 \succ_{a}^{\{a, b\}} 3$; and $1 \succ_{b}^{\{b\}} 3 \succ_{b}^{\{b\}} 2$ and $3 \succ_{b}^{\{a, b\}} 1 \succ_{b}^{\{a, b\}}$ 2. These priorities contain some "acyclicities" depending on which objects are left in the economy. In other words, certain "acyclicities" in the definition of priorities are compatible with consistency, strategy-proofness, and efficiency. Unfortunately, we do not know more about the precise structure of these acyclicities and rules based on such priorities (apart from the rules based on Example 2).
} 
In real life agents may be indifferent between several objects because, for instance, they do not have enough information to distinguish any two of them. Unfortunately, our result (and Theorem 1 of Ergin, 2002) is not robust when allowing for indifferences. The reason is as follows. It is easy to see that any strategy-proof and consistent rule satisfies non-bossiness. Then, on the domain of weak preferences any rule of Theorem 1 satisfies strategy-proofness, non-bossiness, and efficiency. By Bogomolnaia, Deb and Ehlers (2005, Theorem 4) such a rule is "almost" a serial dictatorship. Therefore, on the domain of weak preferences, any rule that satisfies consistency, strategy-proofness, and efficiency must be "almost" a serial dictatorship.

Ehlers and Klaus (2005) consider the richer model of house allocation with quotas, i.e., possibly multiple copies of objects may be available. We could adjust our model accordingly without affecting the results, but chose for the clarity and notational simplicity of the classical house allocation model.

\section{Appendix}

\section{Proof of Theorem 1}

To prove Theorem 1 let $\varphi$ be a rule satisfying consistency, strategy-proofness, and efficiency.

For each object we construct a generalized priority ordering. Given $x \in K$, let $R_{i}^{x} \in \mathcal{R}$ denote a preference relation such that for all $y \in K \backslash\{x\}, x P_{i}^{x} 0 P_{i}^{x} y$, i.e., $x$ is the unique object that is preferred to the null object at $R_{i}^{x}$. Then

$$
i \succ_{x} j \Leftrightarrow \text { for all } H \in \mathcal{H} \text { such that } x \in H, \varphi_{i}\left(\left(R_{i}^{x}, R_{j}^{x}\right), H\right)=x \text {. }
$$

The difficulty of the proof is to show that $\succ_{x}$ is complete whenever necessary in the definition of a generalized priority ordering. Example 1 proves that there may exist agents $i, j$ such that neither $i \succ_{x} j$ nor $j \succ_{x} i$.

Lemma 1. Let $x \in K$ and $N=\{1,2,3,4\} \subseteq P$. If $\varphi_{1}\left(R_{N}^{x},\{x\}\right)=x$, then $1 \succ_{x} 2,1 \succ_{x} 3$, and $1 \succ_{x} 4$.

Proof. Let

$$
\varphi_{1}\left(R_{N}^{x},\{x\}\right)=x .
$$

In order to prove $1 \succ_{x} 2,1 \succ_{x} 3$, and $1 \succ_{x} 4$, we show that for all $H \in \mathcal{H}$ such that $x \in H$, $\varphi_{1}\left(R_{N}^{x}, H\right)=x$.

Let $y \in K \backslash\{x\}$. We first show that

$$
\varphi_{1}\left(R_{N}^{x},\{x, y\}\right)=x
$$

The proof involves many different profiles, which we depict as explained after the two first profiles we introduce now. Recall that by Remark 1, for any economy it suffices to specify the preferences over the objects that are present in that economy. Without loss of generality, we assume

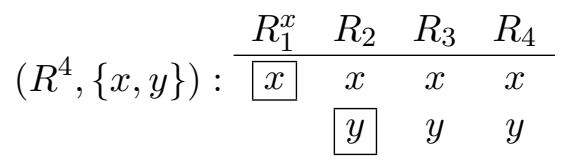


and

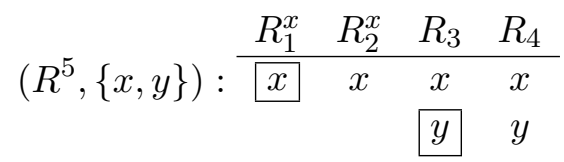

We only specify the ranking of the objects which are preferred to the null object. In (4), the set of objects is $\{x, y\}$ and the rule $\varphi$ allocates $x$ to agent 1 and $y$ to agent 2. Furthermore, we denote the profile in (4) by $R^{4}$. In (4) agent 1 has to receive $x$ because otherwise we obtain a contradiction from (2) and consistency (the agent who receives $y$ in (4) can leave the economy in (2) and (4)). Then by efficiency, one of the agents 2, 3, or 4 receives $y$. Without loss of generality, we suppose that agent 2 receives $y$. Similar arguments yield $\varphi_{1}\left(R^{5},\{x, y\}\right)=x$.

From now on we assume that (3) is not true, i.e., $\varphi_{1}\left(R_{N}^{x},\{x, y\}\right) \neq x$.

Step 1: If $\varphi_{1}\left(R_{N}^{x},\{x, y\}\right) \neq x$, then

$$
\left(R^{6},\{x, y\}\right): \begin{array}{cccc}
R_{1} & R_{2}^{x} & R_{3}^{x} & R_{4}^{x} \\
\hline x & x & x & x \\
y & & &
\end{array}
$$

Because $\varphi_{1}\left(R_{N}^{x},\{x, y\}\right) \neq x$, strategy-proofness implies that in the above economy agent 1 cannot receive $x$. Then by efficiency, $\varphi_{1}\left(R^{6},\{x, y\}\right)=y$. We have to show that neither agent 2 nor agent 3 receives $x$ at $\left(R^{6},\{x, y\}\right)$. Suppose

$$
\left(R^{7},\{x, y\}\right): \begin{array}{cccc}
R_{1} & R_{2}^{x} & R_{3}^{x} & R_{4}^{x} \\
\hline x & x & x & x \\
y & & &
\end{array}
$$

Then consistency and strategy-proofness imply

$$
\left(R^{8},\{x, y\}\right): \begin{array}{cc}
R_{1} & R_{2} \\
\hline x & x \\
y & y
\end{array}
$$

Using consistency and strategy-proofness in (4) yields that agent 1 receives $x$ in the economy (8), a contradiction. Hence, $\varphi_{2}\left(R^{6},\{x, y\}\right) \neq x$.

Suppose

$$
\left(R^{9},\{x, y\}\right): \begin{array}{cccc}
R_{1} & R_{2}^{x} & R_{3}^{x} & R_{4}^{x} \\
\hline x & x & x & x \\
y & & &
\end{array}
$$

Then consistency and strategy-proofness imply

$$
\left(R^{10},\{x, y\}\right): \begin{array}{cc}
R_{1} & R_{3} \\
\hline x & x \\
y & y
\end{array}
$$

Using consistency and strategy-proofness in (5) yields that agent 1 receives $x$ in the economy $(8)$, a contradiction. Hence, $\varphi_{3}\left(R^{6},\{x, y\}\right) \neq x$. Since agents 1,2 , and 3 do not receive $x$ in the economy (6), efficiency implies that 4 receives $x$ in the economy (6). 
Step 2: We finish the proof by showing that in the economy $\left(R^{11},\{x, y\}\right) \in \mathcal{E}^{\{2,3,4\}}$ any efficient allocation yields a contradiction to our previous deductions. Let

$$
\left(R^{11},\{x, y\}\right): \begin{array}{ccc}
R_{2} & R_{3} & R_{4} \\
\hline x & x & y \\
y & y & x
\end{array}
$$

We distinguish three cases.

Case 1: $\varphi_{4}\left(R^{11},\{x, y\}\right)=y$.

Then $\varphi_{2}\left(R^{11},\{x, y\}\right)=0$ or $\varphi_{3}\left(R^{11},\{x, y\}\right)=0$. If $\varphi_{2}\left(R^{11},\{x, y\}\right)=0$, then $\varphi_{3}\left(R^{11},\{x, y\}\right)=x$ and by consistency and strategy-proofness, $\varphi_{2}\left(R_{\{2,4\}}^{y},\{y\}\right)=0$ and $\varphi_{4}\left(R_{\{2,4\}}^{y},\{y\}\right)=y$.

Applying consistency to (4) yields $\varphi_{2}\left(R_{\{2,4\}}^{4},\{y\}\right)=y$ and $\varphi_{4}\left(R_{\{2,4\}}^{4},\{y\}\right)=0$. Thus, by strategy-proofness, $\varphi_{2}\left(R_{\{2,4\}}^{y},\{y\}\right)=y$ and $\varphi_{4}\left(R_{\{2,4\}}^{y},\{y\}\right)=0$, a contradiction.

If $\varphi_{3}\left(R^{11},\{x, y\}\right)=0$, then we obtain a contradiction by applying consistency for agents 3 and 4 to the economies $\left(R^{11},\{x, y\}\right)$ and $\left(R^{5},\{x, y\}\right)$ (in (5)). Hence, Case 1 cannot occur.

Case 2: $\varphi_{4}\left(R^{11},\{x, y\}\right)=x$.

Then $\varphi_{2}\left(R^{11},\{x, y\}\right)=y$ or $\varphi_{3}\left(R^{11},\{x, y\}\right)=y$. If $\varphi_{2}\left(R^{11},\{x, y\}\right)=y$, then agents 2 and 4 gain from exchanging their objects, a contradiction to efficiency. If $\varphi_{3}\left(R^{11},\{x, y\}\right)=y$, then agents 3 and 4 gain from exchanging their objects, a contradiction to efficiency. Hence, Case 2 cannot occur.

Case 3: $\varphi_{4}\left(R^{11},\{x, y\}\right)=0$.

Then, by efficiency, $\varphi_{2}\left(R^{11},\{x, y\}\right)=x$ or $\varphi_{3}\left(R^{11},\{x, y\}\right)=x$. If $\varphi_{2}\left(R^{11},\{x, y\}\right)=x$, then by consistency and strategy-proofness, $\varphi_{2}\left(R_{\{2,4\}}^{x},\{x\}\right)=x$ and $\varphi_{4}\left(R_{\{2,4\}}^{x},\{x\}\right)=0$. tion.

Applying consistency to (6) yields $\varphi_{2}\left(R_{\{2,4\}}^{x},\{x\}\right)=0$ and $\varphi_{4}\left(R_{\{2,4\}}^{x},\{x\}\right)=x$, a contradic-

If $\varphi_{3}\left(R^{11},\{x, y\}\right)=x$, then we obtain a contradiction by applying consistency for agents 3 and 4 to the economies $\left(R^{11},\{x, y\}\right)$ and $\left(R^{6},\{x, y\}\right)$ (in (6)). Hence, Case 3 cannot occur.

We have shown that $\varphi_{1}\left(R_{N}^{x},\{x, y\}\right)=x$. Finally, in order to prove that for all $H \in \mathcal{H}$ such that $x \in H, \varphi_{1}\left(R_{N}^{x}, H\right)=x$, we repeat the previous arguments by adding another object $z \in H \backslash\{x, y\}$ and considering similar preference profiles where $y$ is an undesirable object, etc. $\square$

Next, we show that $\succ_{x}$ is a generalized priority ordering.

Lemma 2. For all $x \in K, \succ_{x}$ is transitive and antisymmetric; i.e., $\succ_{x}$ satisfies condition (i) in the definition of a generalized priority ordering.

Proof. Antisymmetry follows from the definition of $\succ_{x}$.

Let $1,2,3 \in P$. Suppose $1 \succ_{x} 2$ and $2 \succ_{x} 3$. Let $H \in \mathcal{H}$ be such that $x \in H$. In order to prove $1 \succ_{x} 3$, we need to show that $\varphi_{1}\left(\left(R_{1}^{x}, R_{3}^{x}\right), H\right)=x$. Consider $R^{x}=\left(R_{1}^{x}, R_{2}^{x}, R_{3}^{x}\right)$. By efficiency, for some $l \in\{1,2,3\}, \varphi_{l}\left(R^{x}, H\right)=x$.

If $l=2$, then by consistency, $\varphi_{1}\left(\left(R_{1}^{x}, R_{2}^{x}\right), H\right)=0$ and $\varphi_{2}\left(\left(R_{1}^{x}, R_{2}^{x}\right), H\right)=x$, which contradicts $1 \succ_{x} 2$ and the definition of $\succ_{x}$.

If $l=3$, then by consistency, $\varphi_{2}\left(\left(R_{2}^{x}, R_{3}^{x}\right), H\right)=0$ and $\varphi_{3}\left(\left(R_{2}^{x}, R_{3}^{x}\right), H\right)=x$, which contradicts $2 \succ_{x} 3$ and the definition of $\succ_{x}$. 
Thus, $l=1$. By consistency, $\varphi_{1}\left(\left(R_{1}^{x}, R_{3}^{x}\right), H\right)=x$ and $\varphi_{3}\left(\left(R_{1}^{x}, R_{3}^{x}\right), H\right)=0$. Hence, by the definition of $\succ_{x}, 1 \succ_{x} 3$.

In order to determine the set of agents $Q^{x}$ for $\succ_{x}$, we define the following ordering:

$$
i \succ_{x}^{\prime} j \Leftrightarrow \varphi_{i}\left(R_{\{i, j\}}^{x},\{x\}\right)=x .
$$

Note that by efficiency we either have $i \succ_{x}^{\prime} j$ or $j \succ_{x}^{\prime} i$, but not both. Thus, $\succ_{x}^{\prime}$ is complete. The proof that $\succ_{x}^{\prime}$ is transitive is similar to the proof of Lemma 2 .

Let $Q^{x} \subseteq P$ denote the set of the three bottom ranked agents under $\succ_{x}^{\prime}$, i.e., for all $i \in P \backslash Q^{x}$ and all $j \in Q^{x}, i \succ_{x}^{\prime} j$ and $\left|Q^{x}\right|=3$.

Lemma 3. For all $x \in K, \succ_{x}$ satisfies condition (ii) in the definition of a generalized priority ordering.

Proof. Let $Q^{x}=\{l, m, q\}$.

Let $i, j \in P \backslash Q^{x}$. Then by completeness of $\succ_{x}^{\prime}, i \succ_{x}^{\prime} j$ or $j \succ_{x}^{\prime} i$. Suppose $i \succ_{x}^{\prime} j$. By the definition of $Q^{x}, i \succ_{x}^{\prime} l$ and $i \succ_{x}^{\prime} m$. Thus, by consistency, $\varphi_{i}\left(R_{\{i, j, l, m\}}^{x},\{x\}\right)=x$. By Lemma 1 (with $i=1$ and $\{i, j, l, m\}=\{1,2,3,4\}$ ), $i \succ_{x} j$. Hence, $\succ_{x \mid P \backslash Q^{x}}$ is complete and $\succ_{x}$ satisfies (a) in (ii).

Let $i \in P \backslash Q^{x}$. Then by definition of $\succ_{x}^{\prime}$ and consistency, $\varphi_{i}\left(R_{\{i, l, m, q\}}^{x},\{x\}\right)=x$. By Lemma 1 (with $i=1$ and $\{i, j, l, m\}=\{1,2,3,4\}$ ), $i \succ_{x} l, i \succ_{x} m$, and $i \succ_{x} q$. Hence, $\succ_{x}$ satisfies (b) in (ii).

Let $\succ \equiv\left(\succ_{x}\right)_{x \in K}$. Then, $\succ$ is a generalized priority structure.

Lemma 4. The rule $\varphi$ adapts to the generalized priority structure $\succ$.

Proof. Let $N \in \mathcal{P}$ and $(R, H) \in \mathcal{E}^{N}$. Suppose that there exist $x \in H$ and $i \in N$ such that $\varphi(R, H)$ violates the priority of $i$ for $x$. Then for some $j \in N, \varphi_{j}(R, H)=x, i \succ_{x} j$, and $x P_{i} \varphi_{i}(R, H)$. Let $\bar{R} \in \mathcal{R}^{N}$ be such that (i) for all $l \in N \backslash\{i\}$ and all $y \in K \backslash\left\{\varphi_{l}(R, H)\right\}$, $\varphi_{l}(R, H) \bar{R}_{l} 0 \bar{P}_{l} y$ and (ii) for all $y \in K \backslash\{x\}, x \bar{P}_{i} 0 \bar{P}_{i} y$.

Let $l \in N \backslash\{i\}$. By strategy-proofness, $\varphi_{l}\left(\left(\bar{R}_{l}, R_{-l}\right), H\right)=\varphi_{l}(R, H)$. Thus, by consistency, $\varphi\left(\left(\bar{R}_{l}, R_{-l}\right), H\right)=\varphi(R, H)$. Applying the same arguments repeatedly we obtain that $\varphi\left(\left(R_{i}, \bar{R}_{-i}\right), H\right)=\varphi(R, H)$. Let $\bar{H} \equiv H \backslash\left(\cup_{l \in N \backslash\{i, j\}}\left\{\varphi_{l}\left(\left(R_{i}, \bar{R}_{-i}\right), H\right)\right\}\right)$.

By consistency, $\varphi_{i}\left(\left(R_{i}, \bar{R}_{j}\right), \bar{H}\right)=\varphi_{i}(R, H)$ and $\varphi_{j}\left(\left(R_{i}, \bar{R}_{j}\right), \bar{H}\right)=x$. By strategy-proofness and $x P_{i} \varphi_{i}(R, H), \varphi_{i}\left(\bar{R}_{\{i, j\}}, \bar{H}\right)=0$ and $\varphi_{j}\left(\bar{R}_{\{i, j\}}, \bar{H}\right)=x$. Thus, by strategy-proofness, $\varphi_{i}\left(R_{\{i, j\}}^{x}, \bar{H}\right)=0$ and $\varphi_{j}\left(R_{\{i, j\}}^{x}, \bar{H}\right)=x$. By $i \succ_{x} j$ and the definition of $\succ_{x}$, we have for all $x \in H^{\prime} \in \mathcal{H}, \varphi_{i}\left(\bar{R}_{\{i, j\}}, H^{\prime}\right)=x$. Since $x \in \bar{H}$, the previous two facts constitute a contradiction.

By Lemma 4, $\varphi$ is an efficient generalized priority rule. The proof of Theorem 1 is now complete. 


\section{References}

Abdulkadiroğlu, A., and T. Sönmez (1998): "Random Serial Dictatorship and the Core from Random Endowments in House Allocation Problems," Econometrica 66, 689-701.

Abdulkadiroğlu, A., and T. Sönmez (1999): "House Allocation with Existing Tenants," Journal of Economic Theory 88, 233-260.

Abdulkadiroğlu, A., and T. Sönmez (2003): "School Choice: A Mechanism Design Approach," American Economic Review 93, 729-747.

Balinski, M., and T. Sönmez (1999): "A Tale of Two Mechanisms: Student Placement," Journal of Economic Theory 84, 73-94.

Bogomolnaia, A., R. Deb, and L. Ehlers (2005): "Strategy-Proof Assignment on the Full Preference Domain," Journal of Economic Theory, 123, 161-186.

Bogomolnaia, A., and H. Moulin (2001): "A New Solution to the Random Assignment Problem," Journal of Economic Theory 100, 295-328.

Chambers, C.P. (2004): "Consistency in the Probabilistic Assignment Model," Journal of Mathematical Economics 40, 953-962.

Ehlers, L. (2002): "Coalitional Strategy-Proof House Allocation," Journal of Economic Theory 105, 298-317.

Ehlers, L., and B. Klaus (2003): "Resource-Monotonic House Allocation," International Journal of Game Theory 32, 545-560.

Ehlers, L., and B. Klaus (2005): "Efficient Priority Rules," Games and Economic Behavior, forthcoming.

Ehlers, L., B. Klaus, and S. Pápai (2002): "Strategy-Proofness and Population-Monotonicity for House Allocation Problems," Journal of Mathematical Economics 83(3), 329-339.

Ergin, H. İ. (2000): "Consistency in House Allocation Problems," Journal of Mathematical Economics 34, 77-97.

Ergin, H. İ. (2002): "Efficient Resource Allocation on the Basis of Priorities," Econometrica $70,2489-2497$.

Gale, D., and L. S. Shapley (1962): "College Admissions and the Stability of Marriage," American Mathematical Monthly 69, 9-15.

Kesten, O. (2004a): "On two competing mechanisms for priority based allocation problems," Journal of Economic Theory, forthcoming.

Kesten, O. (2004b): "Coalitional Strategy-Proofness and Resource Monotonicity for House Allocation Problems," Working Paper.

Miyagawa, E. (2001): "House Allocation with Transfers," Journal of Economic Theory 100, 329-355. 
Ohseto, S. (2006): "Characterizations of Strategy-Proof and Fair Mechanisms for Allocating Indivisible Goods," Economic Theory, forthcoming.

Pápai, S. (2000): "Strategyproof Assignment by Hierarchical Exchange," Econometrica 68, 1403-1433.

Schummer, J. (2000): "Eliciting Preferences to Assign Positions and Compensation," Games and Economic Behavior 30, 293-318.

Svensson, L.-G. (1999): "Strategy-Proof Allocation of Indivisible Goods," Social Choice and Welfare 16, 557-567.

Svensson, L.-G. (2004): "Strategy-Proof and Fair Wages," Working Paper.

Svenson, L.-G., and B. Larsson (2002): "Strategy-Proof and Nonbossy Allocation of Indivisible Goods and Money," Economic Theory 20, 483-502.

Thomson, W. (2003): On Monotonicity in Economies with Indivisible Goods, Social Choice and Welfare 21, 195-205.

Thomson, W. (2004): “Consistency and its Converse," Working Paper. 